\title{
SIRT7 suppresses the epithelial-to- mesenchymal transition in oral squamous cell carcinoma metastasis by promoting SMAD4 deacetylation
}

\author{
Wenlu Li*, Dandan Zhu and Shuaihua Qin
}

\begin{abstract}
Background: Oral squamous cell carcinoma (OSCC) is one of the most common malignancies and has a poor prognosis. The epithelial-to-mesenchymal transition (EMT) is crucial for increasing the metastasis of OSCC. Recently, studies have indicated that sirtuin7 (SIRT7) is implicated in tumor genesis; however, the potential role of SIRT7 in the EMT and metastasis of OSCC has not been reported.

Methods: We investigated the cellular responses to SIRT7 silencing or overexpression in OSCC cell lines by wound healing assay, migration and invasion assay, western blotting, immunofluorescence and immunohistochemistry.

Results: In the present study, we found that SIRT7 was significantly downregulated in OSCC cell lines and human OSCC/OSCC tissues with lymph node metastasis. Overexpression of SIRT7 decreased the proliferation and invasion of OSCC cells in vitro, whereas SIRT7 knockdown significantly increased OSCC cell growth and invasion. Upregulation of SIRT7 concomitantly increased the expression of E-cadherin, and decreased the expression of mesenchymal markers. SIRT7 overexpression also reduced the level of acetylated SMAD4 in OSCC cells. Moreover, SIRT7 overexpression significantly inhibited OSCC lung metastasis in vivo.

Conclusion: Together, these findings suggested that SIRT7 suppressed EMT in OSCC metastasis by promoting SMAD4 deacetylation.
\end{abstract}

Keywords: sirtuin7, Oral squamous cell carcinoma, Epithelial-to-mesenchymal transition

\section{Background}

Oral cancer is one of the most common life-threatening types of cancer worldwide, with oral squamous cell carcinoma (OSCC) being the predominant form, accounting for over $90 \%$ of all oral malignancies [1-4]. OSCC is associated with high morbidity and mortality $[5,6]$. It has been reported that there are over 300,000 new cases of OSCC and 124,000 deaths per year globally [7]. However, despite treatment advances including multi-agent chemotherapy, radiotherapy, and targeted therapy, the overall 5-year survival rate for OSCC remains disappointingly low $(<50 \%)$, because of its aggressively invasive and metastatic nature, such that most patients with

\footnotetext{
* Correspondence: Iwldoctor@163.com

Department of Stomatology, The First affiliated hospital of Zhengzhou

University, 1\# East Jianshe Road 1, Zhengzhou 450000, Henan, China
}

metastatic disease die within 1 year [8-10]. A better understanding of OSCC pathogenesis and the molecular mechanism underlying its metastasis is therefore needed to inform effective therapies to improve the survival of OSCC patients.

The epithelial-to-mesenchymal transition (EMT) is a vital and fundamental process in the progression and metastasis of OSCC [3, 11-14]. During EMT, epithelial cells gradually lose epithelial structural molecules, polarity, and adhesion capacity, and acquire mesenchymal traits, motility, and invasiveness $[1,15]$. Activation of the EMT results in decreased expression of epithelial markers (E-cadherin and $\beta$-catenin) and increased expression of adhesion and mesenchymal proteins (including vimentin, $\mathrm{N}$-cadherin and fibronectin) $[16,17]$. Transforming growth factor- $\beta$ (TGF- $\beta$ ) signaling is a key regulator of EMT, which is

(C) The Author(s). 2018 Open Access This article is distributed under the terms of the Creative Commons Attribution 4.0 International License (http://creativecommons.org/licenses/by/4.0/), which permits unrestricted use, distribution, and 
mediated by SMAD2, SMAD3, and SMAD4. Among several critical factors involved in the complex progression of the EMT, SMAD4, a central transducer of TGF- $\beta$ signaling, is the most important factor in the clonal evolution and metastases of numerous cancers. Importantly, possible regulators of the SMAD4 pathway in OSCC have rarely been reported, such that further in-depth investigations are merited [18].

Recently, an increasing number of studies have focused on the causal links between aging and cancer metastasis $[19,20]$. Sirtuin7 (SIRT7) is the only intranuclear member of the sirtuin family, and is among the most important regulators of aging and lifespan. As a NAD (+)-dependent deacetylase that participates in ribosome biogenesis and rRNA transcription, SIRT7 has been shown to play a prominent role in metabolism, cell cycle control, and proliferation [21-23]. However, at present there is conflicting evidence regarding the role of SIRT7 in tumor genesis and metastasis [19, 23-27]. Some previous studies have reported high expression of SIRT7 in tumors, and especially in high grade tumors, when compared with normal adjacent control tissue [19, 23, 27]. SIRT7 expression has been shown to be negatively correlated with overall survival in patients with colon carcinoma [28]. In contrast to these results, there is controversy regarding whether SIRT7 is truly a cancer-promoting protein [24-26]. McGlynn et al. reported that SIRT7 possesses tumor-suppressing properties in pancreatic cancer, and low levels of SIRT7 expression were associated with an aggressive tumor phenotype and poorer outcomes [29]. Tang et al. reported that SIRT7 was significantly downregulated in breast cancer lung metastases in humans and mice, which highlighted SIRT7 as a suppressor of breast cancer metastasis [24]. In addition, Lai et al. reported that SIRT7 was significantly downregulated in head and neck squamous cell carcinoma (HNSCC) tissues compared with the adjacent noncancerous tissues [25]. However, no study has reported the effect of SIRT7 on OSCC, so the underlying mechanism of this cancer remains elusive.

The present study investigated the role of SIRT7 in OSCC. We evaluated the expression of SIRT7 in human OSCC tissues. Furthermore, the specific effects of SIRT7 on OSCC cell proliferation, migration, EMT, and metastasis, together with the possible underlying mechanisms, were also characterized for the first time.

\section{Results}

\section{SIRT7 expression is correlated with OSCC progression}

We first determined whether SIRT7 expression was associated with human OSCC progression. Immunohistochemical staining was performed on 20 primary OSCC without lymph node metastasis (P) and 20 OSCC with lymph node metastasis $(\mathrm{M})$, and tumor-adjacent normal oral tissues (NC). Representative photographs are shown in Fig. 1a-b. The results showed that there was a significantly lower level of SIRT7 expression in P and M tissues than in NC tissues, which was also confirmed by Western blot (WB) analysis (Fig. 1c-d). The level of SIRT7 were examined by WB analysis between metastatic lymph nodes tissuess (ML) than the matched primary lymph node (NL). The results revealed that a markly higher in matched primary lymph nodes than in metastatic lymph nodes tissues (Fig. 1e-f). Moreover, WB analysis also showed that SIRT7 protein expression was significantly increased in human OSCC cell lines when compared with HOK cells (Fig. 1g-h). To further determine the relationship between SIRT7 expression and human OSCC progression, a bioinformatics analysis based on the ProgeneV2 prognostic database (http:// www.abren.net/PrognoScan/) was conducted, which showed that SIRT7 expression correlated inversely with the survival of patients with OSCC, although this correlation did not reach statistical significance (Fig. 1g). In addition, to confirm the status of EMT in metastasis and non-metastasis tumors, E-Cadherin and vimentin were evaluated in primary and metastatic mucosa tissues. The WB analysis shown that higher E-Cadherin levels and lower vimentin levels were in $\mathrm{P}$ group compared with those in M group (Fig. 1j-l).

\section{SIRT7 impairs OSCC cell migration and invasion in vitro}

To investigate the effects of SIRT7 in OSCC cell lines, lentiviral vectors were used to overexpress and knockdown SIRT7 expression in HSC3 and OECM1 cell lines. As shown in Fig. 2a-b, WB analysis showed that successful SIRT7 overexpression and knockdown were achieved using lentiviral particles. Because SIRT7 expression is related to OSCC progression, SIRT7 may play important roles in one or more steps of OSCC metastasis. We first examined the effects of SIRT7 on OSCC cell migration and invasion. The results of the wound migration assay showed that the wound-healing abilities of SIRT7-overexpressing OSCC cells were significantly lower than those of the control group, while SIRT7 knockdown markedly increased migration (Fig. 2c-d). We further confirmed these findings using a Transwell ${ }^{\circ}$ invasion assay. Consistent with results obtained from the wound-healing assay, lentiviral shRNA-mediated SIRT7 silencing significantly increased the invasion of OSCC cells. In contrast, SIRT7 overexpression decreased the invasion of OSCC cells (Fig. 2e).

\section{SIRT7 suppresses the EMT in OSCC cells}

To identify the molecular mechanisms involved in SIRT7-mediated regulation of cell migration and invasion in OSCC cell lines, an analysis of the main EMT markers was performed. As shown in Fig. 3a-b, the results of WB analysis showed that SIRT7 overexpression dramatically increased 


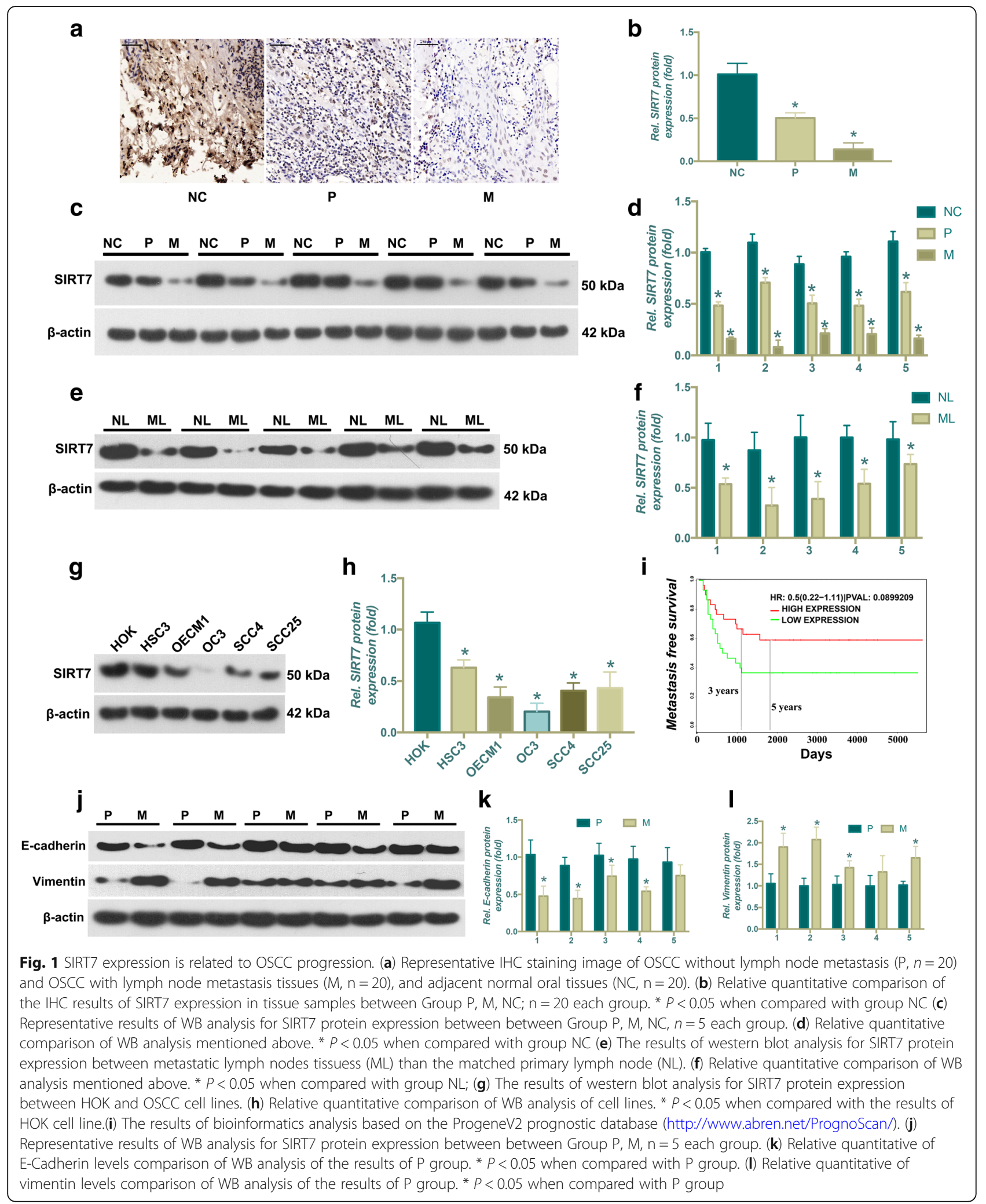

E-cadherin expression and downregulated the protein levels of N-cadherin, vimentin, and MMP7. In contrast, lentiviral shRNA-mediated SIRT7 silencing significantly decreased
E-cadherin expression and upregulated the protein levels of $\mathrm{N}$-cadherin, vimentin, and MMP7. The effects of SIRT7 on $\mathrm{E}$-cadherin and $\mathrm{N}$-cadherin were further confirmed by 

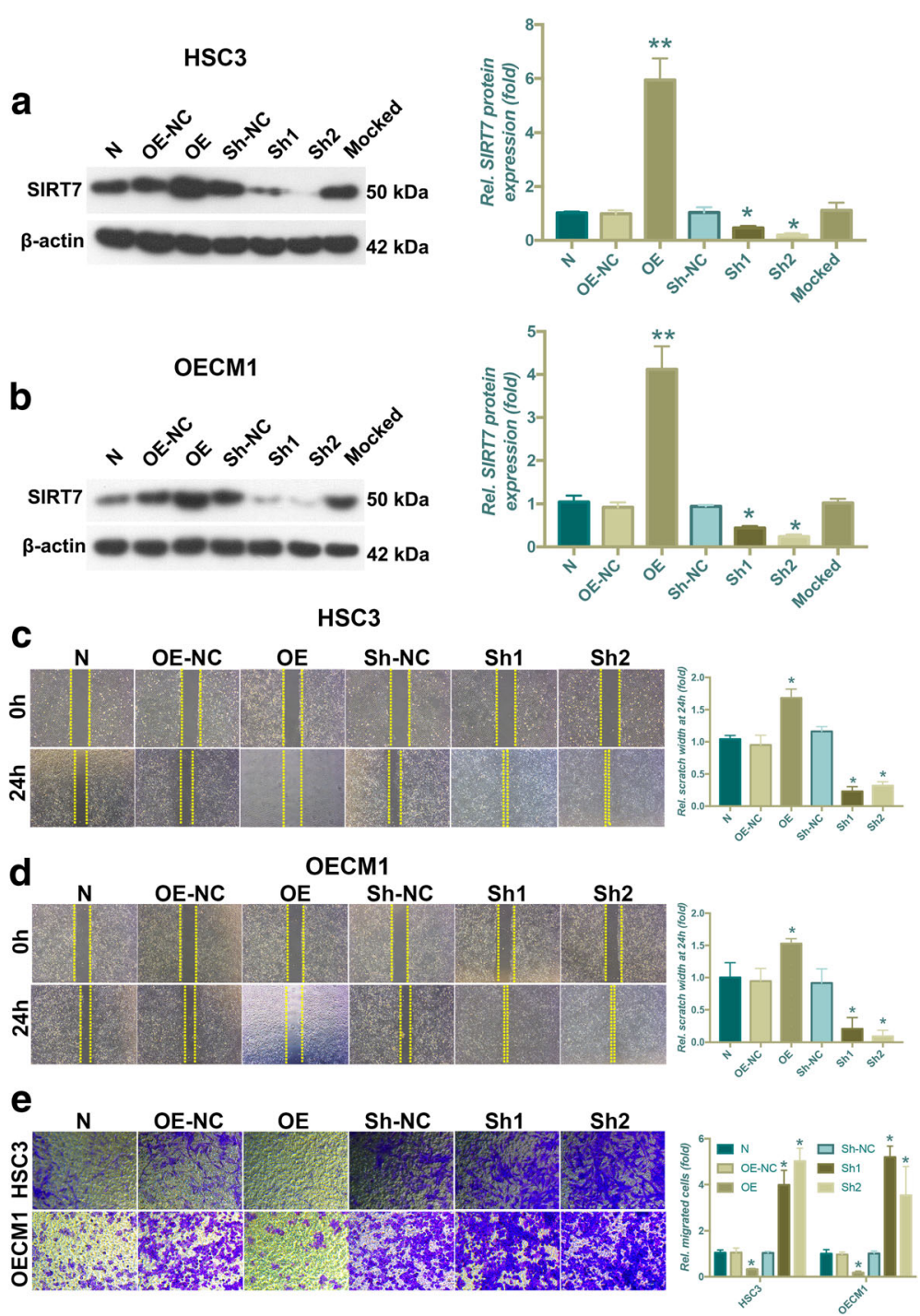

Fig. 2 SIRT7 impairs OSCC cells migration and invasion in vitro. (a) The results of western blot analysis and its quantitative analysis showed successful upregulation and downregulation of SIRT7 levels in HSC3 cells using lentiviral particles. (b) The results of western blot analysis and its quantitative analysis showed successful upregulation and downregulation of SIRT7 levels in OECM1 cells using lentiviral particles. (c) The results of wound migration assay and quantitative analysis of the effect SIRT7 on HSC3 cells migration. (d) The results of wound migration assay and quantitative analysis of the effect SIRT7 on OECM1 cells migration. (e) The results of transwell invasion assay and quantitative analysis of the effect SIRT7 on HSC3 and OECM1 cells invasion. N: normal control; OE: overexpression; Sh: shRNA that used for SIRT7 knockdown; NC: negative control. * $P<0.05$ when compared with group $N$

immunofluorescence staining (Fig. 3c-d). Taken together, these results showed that SIRT7 overexpression suppressed the EMT in OSCC cells.

\section{SIRT7 suppresses the EMT in OSCC cells by deacetylating SMAD4}

To identify the molecular mechanism underlying the effects of SIRT7 on the EMT in OSCC cells, components of the SMAD4 signaling pathway, a key regulator of the EMT, were evaluated. As shown in Fig. 4a, WB analysis showed that SIRT7 overexpression significantly promoted SMAD4 deacetylation; the acetylated (Ac)-SMAD4 protein expression level was decreased during SIRT7 overexpression. In contrast, SIRT7 knockdown increased the protein level of Ac-SMAD4, which was further confirmed by immunofluorescence staining (Fig. 4b). After application of a siRNA that targeted SMAD4, the effect of SIRT7 knockdown on increasing Ac-SMAD4 expression was significantly reduced. In addition, the effect of SIRT7 knockdown on increasing the EMT in OSCC cells was also significantly inhibited (Fig. 4c-d). Taken together, these results suggested that the suppressive effect of SIRT7 on the EMT in OSCC cells was at least partially mediated by promotion of SMAD4 deacetylation. 


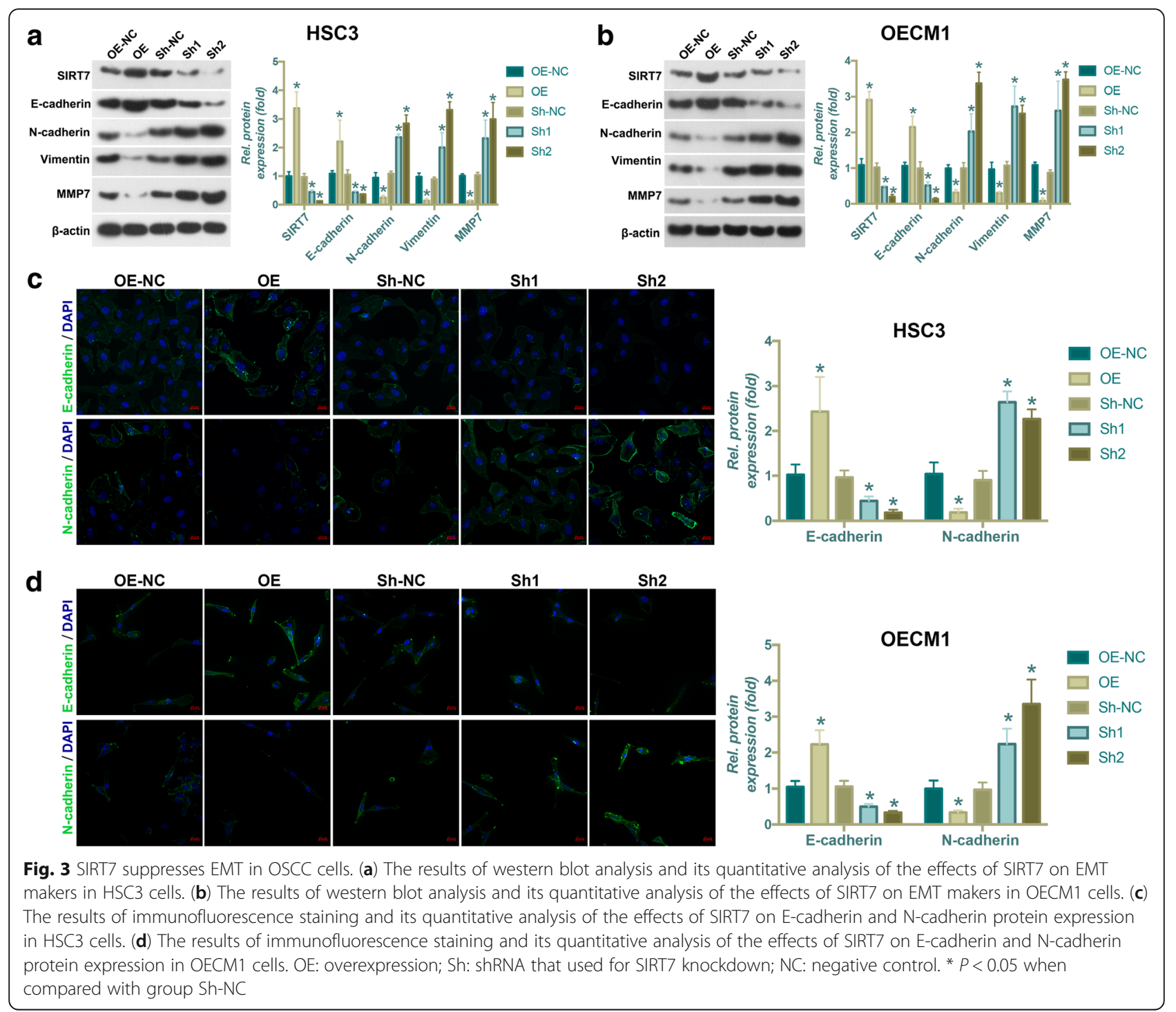

\section{SIRT7 overexpression inhibits metastasis in vivo}

The in vivo anti-metastatic effects of SIRT7 on OSCC were determined using a tail vein metastatic assay. Compared with mice injected with SIRT7-overexpressing HSC 3 and OECM1 cells, more nodules in the lung were observed in mice that were injected with empty virus vector-infected HSC3 and OECM1 cells (Fig. 5a-b). Hematoxylin and eosin staining showed that tail vein injection with cells stably overexpressing SIRT7 led to significantly fewer and smaller nodules in lungs compared with the control group (Fig. $5 \mathrm{c}-\mathrm{d}$ ). In addition, increased $\mathrm{E}$-cadherin and downregulated $\mathrm{N}$-cadherin, vimentin, and MMP7 mRNA levels were observed in the lung tissue of the SIRT7 overexpression group. Taken together, these results showed the remarkable inhibitory effects of SIRT7 overexpression on pulmonary metastasis of OSCC cells.

\section{Discussion}

We have shown that SIRT7, a newly discovered and pivotal regulator of lifespan and aging, acted as a tumor metastasis-suppressing gene in OSCC, as shown by its downregulation promoting OSCC cell line invasion and migration (whereas its overexpression suppressed metastasis). The decreased expression of SIRT7 in primary OSCC tissues without metastatic lymph node and OSCC tissues with metastatic lymph node indicated that it may be useful for predicting the prognosis of OSCC patients, as well as the likelihood of relapse and metastasis. We further found that SIRT7 overexpression-induced metastasis suppression was dependent on its negative regulation of the EMT, at least partially through promoting SMAD4 deacetylation, because its upregulation induced resistance to the EMT, whereas its deletion attenuated the EMT and metastasis in OSCC. 

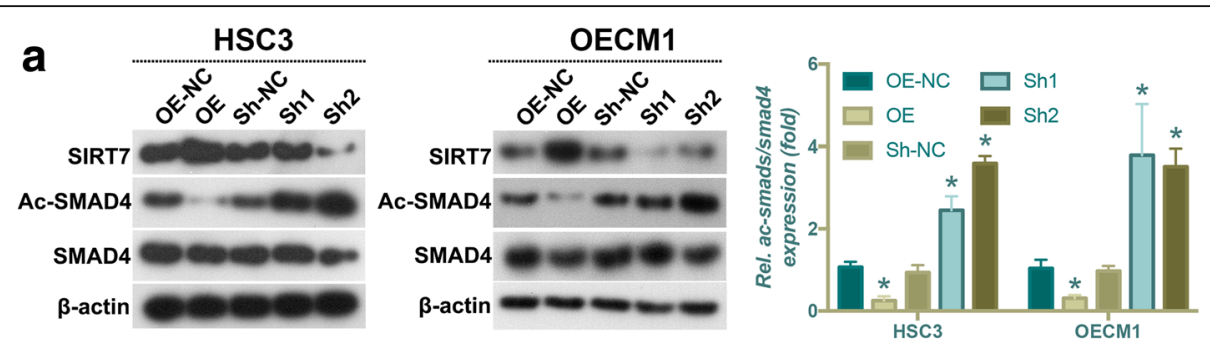

b
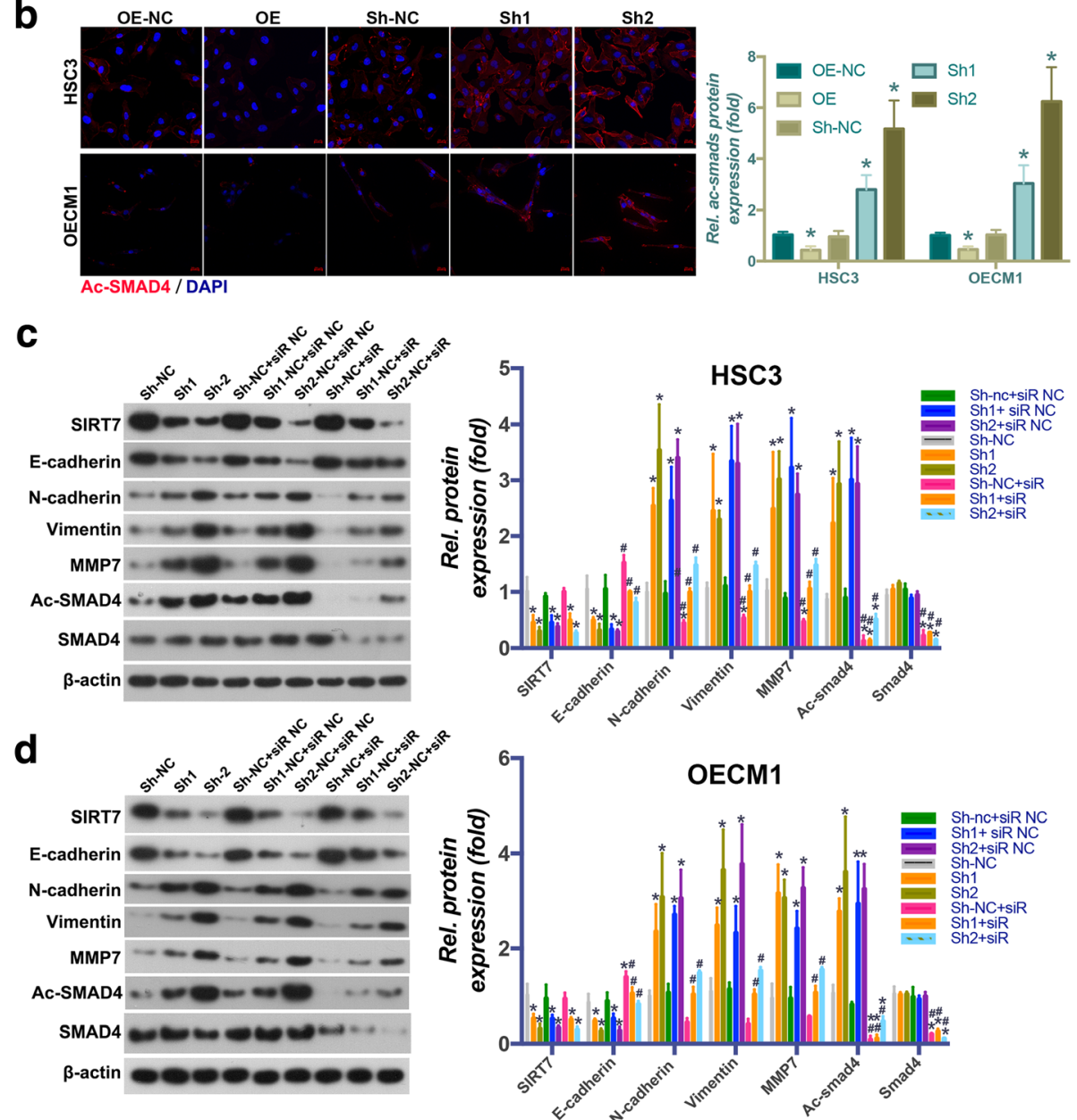

Fig. 4 SIRT7 suppresses EMT in OSCC cells through deacetylating SMAD4. (a) The results of western blot analysis and its quantitative analysis of the effects of SIRT7 on SMAD4 and Ac-SMAD4 protein expression in HSC3 and OECM1 cells. (b) The results of immunofluorescence staining and its quantitative analysis of the effects of SIRT7 on Ac-SMAD4 protein expression in HSC3 and OECM1 cells. (c) The results of western blot analysis and its quantitative analysis of the effects of SIRT7 on SMAD4, AC-SMAD4 and EMT markers protein expression in HSC3 cells. (d) The results of western blot analysis and its quantitative analysis of the effects of SIRT7 on SMAD4, Ac-SMAD4 and EMT markers protein expression in OECM1 cells. OE: overexpression; Sh: shRNA that used for SIRT7 knockdown; NC: negative control. * $P<0.05$ when compared with group OE-NC; $\# P<0.05$ when compared with group siR NC groups

This study emphasized the novel and necessary role of SIRT7 in tumor metastasis. Although previous reports studied the role and function of SIRT7 in various cancers, they mainly focused on tumor growth (e.g., cell proliferation, apoptosis, and cell cycle arrest). Furthermore, the results of these studies were controversial: some reported that SIRT7 was responsible for the maintenance of oncogenic transformation via H3K18ac deacetylation [30]. $\mathrm{Yu}$ et al. reported that overexpression of SIRT7 had oncogenic properties and served as a prognostic factor in colorectal cancer [28]. Similar results were also reported by Malik et al. [31]. However, Vakhrusheva et al. reported that as an anti-aging factor, SIRT7 was consistently downregulated in tumorigenic cells. SIRT7 is thought to play an 
a

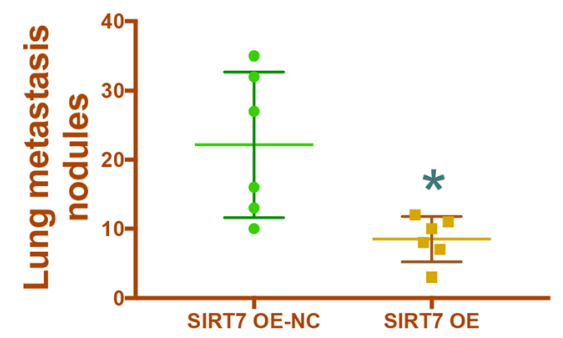

b

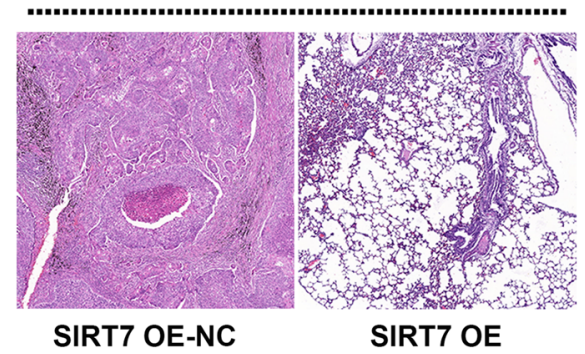

HSC3
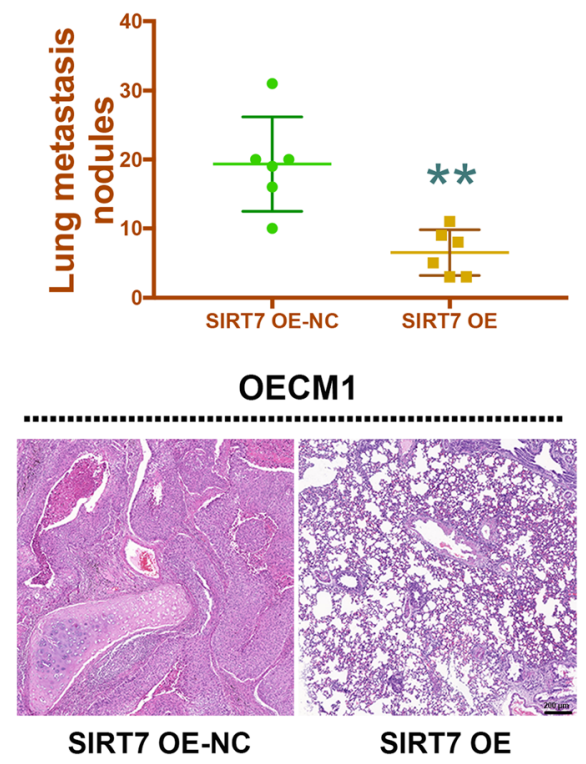

C
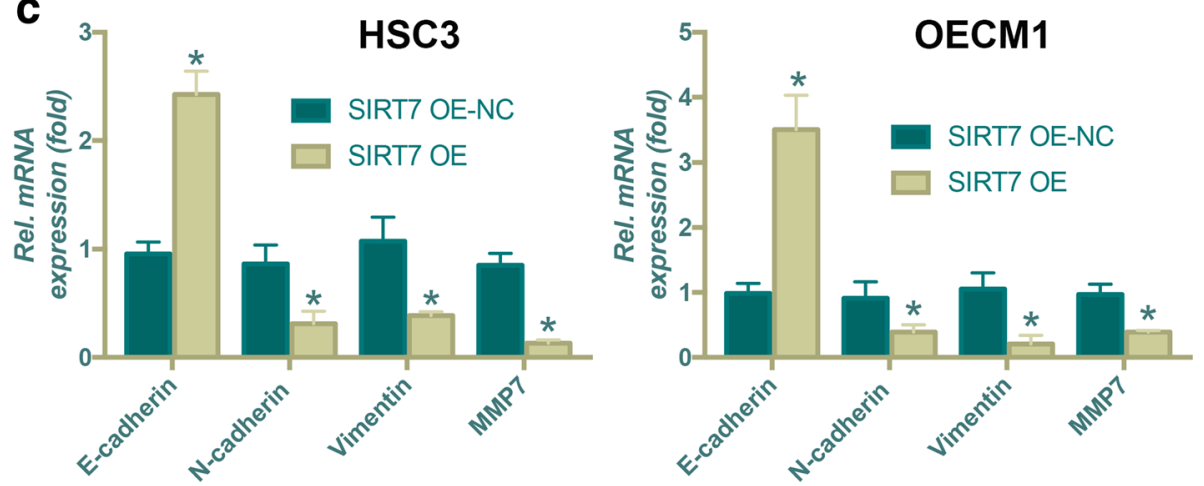

Fig. 5 SIRT7 overexpression inhibits metastasis in vivo. (a) Scatter plot showing lung metastatic nodules ( $n=6$ for each group). (b). Representative hematoxylin and eosin (HE) images for OSCC metastatic lung nodules. (c) mRNA levels of E-cadherin, N-cadherin, Vimentin, and MMP7 of each group. OE: overexpression; NC: negative control. ${ }^{*} P<0.05$ when compared with group OE-NC

important role in mediating tissue integrity during aging by inhibiting cell growth and proliferation [32]. Lai et al. analyzed SIRT genes in human HNSCC tissues (including OSCC) from 39 patients and reported that SIRT7 was negatively correlated with the progression of human HNSCC [25]. Because of these controversial findings, Tang et al. [24] conducted an in depth study of breast cancer and reported that SIRT7 may itself be dynamically regulated during tumor development, which means that at an early stage, high SIRT7 could contribute to oncogenic transformation and tumor growth while, later, it inhibits migration and invasion of tumor cells. Although the results of our study were consistent with their findings, future studies focusing on dynamic changes in SIRT7 role during tumorigenesis are still required.

There is presently little known about the potential role of SIRT7 in cancer metastasis. Our results showed that upregulation of SIRT7 significantly impaired the invasion and migration of OSCC, both in vitro and in vivo, but its knockdown dramatically promoted OSCC metastasis. A recent report by Tang et al. [24] also indicated that upregulation of SIRT7 antagonized the EMT of breast cancer and inhibited its metastasis. To some extent, this study further supported our findings that SIRT7 plays an important role in tumor invasion, migration, and metastasis.

The EMT has widely been considered as a vital process in regulating the initial steps in OSCC metastasis. Because of its clinical importance, suppression of the EMT is becoming an attractive therapeutic strategy that can significantly improve the disease outcome of cancer patients. Our findings implicated SIRT7 as a key regulator of the EMT in OSCC, where overexpression of SIRT7 induced a range of biochemical (increased E-cadherin and decreased $\mathrm{N}$-cadherin and vimentin) and 
functional (decreased MMP9 production, and decreased invasion and migration capacity) changes. TGF- $\beta$ signaling is a key regulator of the EMT, which is mainly mediated by SMAD4. However, little is known regarding the possible correlation between the regulation of SMAD4 and the EMT of OSCC. Consistent with previous studies, we found that SIRT7 deacetylated and promoted SMAD4 degradation [24]. In another study, Chen et al. reported that by deacetylating SMAD4, the SIRT1 enzyme can also reduce MMP7 expression and consequently inhibit cell migration, invasion, and tumor metastasis in OSCC [18].

Loss of SIRT1 has been reported to cause hyper-acetylation of SMAD4 and promote breast cancer metastasis [33]. Together with recently reported studies about the interaction between SIRT1 and SIRT7 during gene transcription [31], the impact of SIRT7 on the EMT may also be closely regulated by SIRT1, but further studies are needed to confirm this possibility.

Although additional future studies are needed to identify the detailed mechanism, our results indicated that SIRT7 suppressed the EMT in OSCC metastasis by promoting SMAD4 deacetylation, which constitutes an important insight into the role of SIRT7 in tumor metastasis.

\section{Methods}

\section{Clinical tissues}

This study was approved by the Medical Ethics Committee of the First affiliated hospital of Zhengzhou University Hospital. All subjects provided written informed consent, and none of them received chemo radiotherapy prior to surgery. Clinical tissue samples of OSCC and the paired adjacent normal tissue, together with lymph node metastasis samples were obtained during surgery. The relevant clinical information were in Table 1.

Table 1 The clinical profiles of the 40 patients with OSCC

\begin{tabular}{llll}
\hline Clinical & No. of patients & & $P$ value \\
\cline { 2 - 3 } parameters & Metastatic $(n=20)$ & Nonmetastatic $(n=20)$ & \\
\hline Gender & 12 & 10 & $>0.05$ \\
Male & 8 & 10 & \\
Age & & & $>0.05$ \\
mean \pm SD & $51.23 \pm 11.63$ & $56.82 \pm 15.59$ & \\
Lymph node status & 0 & & $<0.001$ \\
N- & 20 & 20 & \\
N+ & 20 & 0 & \\
Tumor classification & 13 & \\
T1 + T2 & 2 & 7 & \\
T3 + T4 & 18 & & \\
\hline
\end{tabular}

\section{Bioinformatics analysis}

The ProgeneV2 prognostic database (http://www.abren.net/PrognoScan/) was used to collect information for analysis of the effect of SIRT7 on survival in OSCC patients [34, 35]. Kaplan-Meier curve was applied for analyzing survival rate of patients with $\mathrm{OC}$.

\section{Immunohistochemistry (IHC)}

IHC staining was performed in paraffin-embedded specimens a blind manner as follows: the slides were incubated overnight with rabbit anti-SIRT7 monoclonal antibody (1:100; Abcam, Cambridge, UK) employing an avidin-biotin complex method. A secondary antibody was then applied for $30 \mathrm{~min}$ at room temperature.

\section{Cell culture}

The HOK cells and five human OSCC cell lines (HSC3, OECM1, OC3, SCC4, and SCC25 used in this study were purchased commercially from the American Type Culture Collection (ATCC; Manassas, VA, USA). The HOK cells were cultured in oral keratinocyte growth medium (ScienCell, Carlsbad, CA, USA), HSC-3 and OC3 cells were cultured in DMEM) medium, OECM-1 cells were maintained in RPMI 1640 medium, while SCC4 and SCC25 cells were cultured in DMEM/F12 medium. All the medium mentioned above were supplemented with $10 \%$ fetal bovine serum (FBS; Hyclone, Israel) and 100 units $/ \mathrm{mL}$ penicillin and streptomycin $(\mathrm{P} /$ $\mathrm{S}$, Invitrogen, Camarillo, CA, USA). Cells were maintained at $37^{\circ} \mathrm{C}$ in a atmosphere filled with $5 \% \mathrm{CO} 2$.

\section{Immunofluorescence staining}

For Immunofluorescence staining, cells were grown onto coverslips and fixed in 4\% paraformaldehyde for $10 \mathrm{~min}$, and blocked with in $0.05 \%$ Triton X-100 and 3\% bovine serum albumin (BSA) for $30 \mathrm{~min}$. The coverslips were incubated primary antibodies (N-cadherin (1:100; Abcam, Cambridge, United Kingdom), E-cadherin (1:100; Abcam, Cambridge, United Kingdom), acetylated smad4 (1:100; Proteintech, Wuhan, China)) overnight at $4{ }^{\circ} \mathrm{C}$, followed by fluorescence-conjugated secondary antibodies (Beyotime) for $2 \mathrm{~h}$ at room temperature, and nuclei were stained with DAPI (Beyotime) for $5 \mathrm{~min}$.

\section{Cell transfection and RNA interference}

A lentiviral short hairpin RNA (shRNA) construct targeting SIRT7 was obtained from Sigma-Aldrich (St. Louis, MO, USA). For stable knockdown, the shRNAs were annealed, and cloned into the pLKO.1 vector (Sigma). The SIRT7 overexpression particles were purchased by GenePharma (Shanghai, China). For retroviral overexpression of SIRT7, polymerase chain reaction (PCR) was used to obtain SIRT7 cDNA, which was then subcloned into the BamHI and XhoI sites of the LV3 
retroviral vector. Lentiviral transfection was performed strictly according to the manufacturer's protocols. In brief, OSCC cells (HSC3 and OECM1 cells) were seeded at $2 \times 10^{5}$ cells/well in a 6 -well plate before transfection and incubated with $2 \mathrm{ml}$ of complete medium for $24 \mathrm{~h}$. Cells were then transfected with lentiviral particles for $12 \mathrm{~h}$, after that the virus-containing medium was replaced with fresh complete medium. Finally, cells were selected using $4 \mu \mathrm{g} / \mathrm{ml}$ puromycin for $96 \mathrm{~h}$ to get OSCC cells with stable SIRT7 overexpression or knock-down. Empty lentiviral vectors were used as a control. siRNA transfection targeting SMAD4 was performed with Lipofectamine 2000 (Thermo) following the manufacturer's instruction, using siRNAs synthesized in GenePharma (Shanghai, China).

\section{RNA isolation and quantitative real-time PCR (RT-qPCR)}

Total RNA was isolated using TRIzol reagent (Takara, Dalian, China), which was then reversely transcribed into cDNA using PrimeScript RT-PCR kit (TaKaRa Biotechnology) according to the protocol. The RT-qPCR was performed using the Power SYBR Green PCR Master Mix on the ABI 7900 Prism HT (Applied Biosystems). Primers used are followed: E-cadherin, Forward: CGAGAGCTACACGT TCACGG; Reverse: GGGTGTCGAGGGAAAAATAGG; N-cadherin, Forward: TTTGATGGAG.

GTCTCCTAACACC; Reverse: ACGTTTAACACGTT GGAAATGTG; Vimentin, Forward: GCCCTAGACGA ACTGGGTC; Reverse: GGCTGCAACTGCCTAATGA.

G; MMP7, Forward: GAGTGAGCTACAGTGGGAA CA; Reverse: CTATGACGC.

GGGAGTTTAACAT; GAPDH, Forward: TGTGGG CATCAATGGATTTGG; Reverse: ACACCATGTATTC CGGGTCAAT. The $2^{-\triangle \Delta C t}$ method was used to calculate relative gene expression.

\section{Wound healing assay}

The wound healing assay was performed to test the effect of SIRT7 in OSCC cell migration. OSCC cells (HSC3 and OECM1 cells) with SIRT7 overexpression and knock-down together with the control groups were cultured in into 12-well plates and cultured to $90 \%$ confluence. The cells were then starved overnight, and the wound was scratched in the center of the cell monolayer using a sterile $1-\mathrm{mL}$ micropipette tip. The wound was photographed under a microscope (DM IL, Leica Microsystems) at indicated time ( $0 \mathrm{~h}$ and $24 \mathrm{~h}$ after the wound was created). Image $J$ sofware was used to quantify the relative migration rate.

\section{Transwell invasion assay}

The transwell invasion assay was performed to test the effect of SIRT7 in OSCC cell invasion. After transfection, $5 \times 10^{4}$ OSCC cells (HSC3 and OECM1 cells) suspended in medium without FBS were incubated on the upper chamber membranes using a BioCoat Matrigel Invasion Chamber (Corning) strictly according to the Cell Invasion Assay protocol. The lower chamber was incubated in $500 \mu \mathrm{l}$ medium with $10 \%$ FBS. To evaluate the invasive ability of OSCC cells, non-invasive cells were removed by cotton swabs and invasive cells were stained with crystal violet and counted under a microscope (DM IL, Leica Microsystems).

\section{Western blot (WB) analysis}

Protein extracts from OSCC cells and clinical tissue samples were prepared in radioimmunoprecipitation assay lysis buffer supplemented with a protease inhibitor (Beyotime, Haimen, China). Protein samples were separated by $10 \%$ SDS polyacrylamide gels and then transferred to a PVDF membrane (Millipore). After blocking in 5\% BSA for $1 \mathrm{~h}$, the membranes were incubated overnight at $4{ }^{\circ} \mathrm{C}$ with primary antibodies specific to $\beta$-actin (1:2000; CST), N-cadherin (1:1000; CST), E-cadherin (1:1000; CST), SIRT7 (1:1000; CST), MMP7(1:1000; CST), SMAD4 (1:1000; CST), Vimentin (1:1000; CST), and acetylated SMAD4 (1:100; Proteintech, Wuhan, China), which was then probed with a horseradish peroxidase-conjugated goat anti-rabbit IgG (1:1000) as a secondary antibody for $2 \mathrm{~h}$ at room temperature. $\beta$-actin was served as the internal control for all western blots. Immunoblots were visualized and quantified using the Bio-Rad system (Bio-Rad, Hercules, CA, USA).

\section{Xenograft model of OSCC lung metastasis}

Animal studies were approved and conducted strictly in accordance with institutional ethical guidelines the Committee on the Use of Live Animals in Teaching and Research of Zhengzhou University. Briefly, 4-week-old pathogen-free female BALB/c-nu mice were purchased from the Slaccas (Shanghai), which were housed in SPF barrier facilities under a $12 \mathrm{~h}$ light/dark cycle, and supplied with sterilized food and water. For in vivo xenograft studies, 24 mice were randomly selected and divided into four groups. On day $0,1 \times 10^{6} \mathrm{HSC}^{\wedge} \mathrm{OE}-\mathrm{NC}, \mathrm{HSC} 3^{\wedge}$ sirt7 $\mathrm{OE}, \mathrm{OECM} 1^{\wedge} \mathrm{OE}-\mathrm{NC}, \mathrm{OECM} 1^{\wedge} \mathrm{SIRT} 7$ OE cells suspended in $0.1 \mathrm{ml}$ of PBS were injected via tail vein of each mouse (six mice in each group). Mice were killed at 8 weeks after tail vein injection, lungs were collected and stored in liquid nitrogen or fixed in formalin for further analysis. The number of metastatic nodules on lung surface was counted under dissecting microscope after hematoxylin and eosin (H\&E) staining.

\section{Statistical analysis}

All experiments were carried out with at least three replicates. The data were shown as mean \pm S.D. One-way ANOVA with Dunnett's test or Newman Keuls test and 
Student's two-tailed t-test were used to calculate the significant differences. Differences were considered as statistically significant when $P<0.05$.

\section{Conclusions}

In conclusion, the present study showed that SIRT7 overexpression suppressed cell migration and invasion in OSCC. Furthermore, SIRT7 suppressed the EMT of OSCC, at least partially by promoting SMAD4 deacetylation. Our study also showed the functional significance of SIRT7 upregulation in metastatic OSCC.

\section{Abbreviation}

EMT: epithelial-to-mesenchymal transition; OSCC: oral squamous cell carcinoma; SIRT7: sirtuin7; SMAD: drosophila mothers against decapentaplegic protein;

TGF- $\beta$ : transforming growth factor- $\beta$; WB: western blot

\section{Availability of data and materials}

Please contact author for data requests.

\section{Authors' contributions}

Conception and design of the research: WL; acquisition of data: DZ; analysis and interpretation of data: SQ; statistical analysis: DZ; drafting the manuscript: WL and DZ; revision of manuscript for important intellectual content: WL. All authors read and approved the final manuscript.

\section{Ethics approval and consent to participate}

All procedures performed in studies involving human participants were in accordance with the ethical standards of the Research Ethics Committee of the First affiliated hospital of Zhengzhou University. All subjects provided written informed consent. Animal studies were approved and conducted strictly in accordance with institutional ethical guidelines the Committee on the Use of Live Animals in Teaching and Research of Zhengzhou University.

\section{Consent for publication}

Not applicable.

\section{Competing interests}

The authors declare that they have no competing interests.

\section{Publisher's Note}

Springer Nature remains neutral with regard to jurisdictional claims in published maps and institutional affiliations.

Received: 9 May 2018 Accepted: 28 June 2018

Published online: 13 July 2018

\section{References}

1. Xie T, Li X, Ye F, Lu C, Huang H, Wang F, Cao X, Zhong C. High KIF2A expression promotes proliferation, migration and predicts poor prognosis in lung adenocarcinoma. Biochem Biophys Res Commun. 2018;497:65-72.

2. Li B, Chen M, Lu M, Xin-Xiang J, Meng-Xiong P, Jun-Wu M. Glutaredoxin 3 promotes migration and invasion via the notch signalling pathway in oral squamous cell carcinoma. Free Radic Res. 2018:1-12.

3. Yang H, Pan L, Xu C, Zhang Y, Li K, Chen S, Zhang B, Liu Z, Wang LX, Chen $H$. Overexpression of tumor suppressor gene ZNF750 inhibits oral squamous cell carcinoma metastasis. Oncol Lett. 2017;14:5591-6.

4. Rao SJ, Rao JBM, Rao PJ. Immunohistochemical analysis of stromal fibrocytes and myofibroblasts to envision the invasion and lymph node metastasis in oral squamous cell carcinoma. J Oral Maxillofac Pathol. 2017; 21:218-23.

5. Zidar N, Bostjancic E, Malgaj M, Gale N, Dovsak T, Didanovic V. The role of epithelial-mesenchymal transition in squamous cell carcinoma of the oral cavity. Virchows Arch. 2017;

6. Wang SH, Chang JS, Hsiao JR, Yen YC, Jiang SS, Liu SH, Chen YL, Shen YY, Chang JY, Chen YW. Tumour cell-derived WNT5B modulates in vitro lymphangiogenesis via induction of partial endothelial-mesenchymal transition of lymphatic endothelial cells. Oncogene. 2017;36:1503-15.

7. MR DEA, Perez-Sayans M, Suarez-Penaranda JM, Somoza-Martin JM, GarciaGarcia A. p27(Kip1) expression as a prognostic marker for squamous cell carcinoma of the head and neck.

8. Li Z, Liu J, Li L, Shao S, Wu J, Bian L, He Y. Epithelial mesenchymal transition induced by the CXCL9/CXCR3 axis through AKT activation promotes invasion and metastasis in tongue squamous cell carcinoma. Oncol Rep. 2018;39:1356-68.

9. Xu Q, Zhang Q, Ishida Y, Hajjar S, Tang X, Shi H, Dang CV, Le AD. EGF induces epithelial-mesenchymal transition and cancer stem-like cell properties in human oral cancer cells via promoting Warburg effect. Oncotarget. 2017:8:9557-71.

10. Tamura T, Ichikawa T, Nakahata S, Kondo Y, Tagawa Y, Yamamoto K, Nagai $K$, Baba T, Yamaguchi R, Futakuchi M, et al. Loss of NDRG2 expression confers oral squamous cell carcinoma with enhanced metastatic potential. Cancer Res. 2017;77:2363-74.

11. Kim SE, Shin SH, Lee JY, Kim CH, Chung IK, Kang HM, Park HR, Park BS, Kim IR. Resveratrol induces mitochondrial apoptosis and inhibits epithelial-Mesenchymal transition in oral squamous cell carcinoma cells. Nutr Cancer. 2018;70:125-35.

12. Wang H, Deng X, Zhang J, Ou Z, Mai J, Ding S, Huo S. Elevated expression of zinc finger protein 703 promotes cell proliferation and metastasis through PI3K/AKT/GSK-3beta Signalling in oral squamous cell carcinoma. Cell Physiol Biochem. 2017;44:920-34

13. Schwock J, Bradley G, Ho JC, Perez-Ordonez B, Hedley DW, Irish JC, Geddie WR. SNAI1 expression and the mesenchymal phenotype: an immunohistochemical study performed on 46 cases of oral squamous cell carcinoma. BMC Clin Pathol. 2010;10(1)

14. Huber MA, Maier HJ, Alacakaptan M, Wiedemann E, Braunger J, Boehmelt G, Madwed JB, Young ER, Marshall DR, Pehamberger H, et al. BI 5700, a selective chemical inhibitor of IkappaB kinase 2, specifically suppresses epithelial-Mesenchymal transition and metastasis in mouse models of tumor progression. Genes Cancer. 2010;1:101-14.

15. Lu M, Wang C, Chen W, Mao C, Wang J. miR-654-5p targets GRAP to promote proliferation, metastasis, and Chemoresistance of oral squamous cell carcinoma through Ras/MAPK signaling. DNA Cell Biol. 2018;

16. Chung MK, Jung YH, Lee JK, Cho SY, Murillo-Sauca O, Uppaluri R, Shin JH, Sunwoo JB. CD271 confers an invasive and metastatic phenotype of head and neck squamous cell carcinoma through the Upregulation of slug. Clin Cancer Res. 2018;24:674-83.

17. Zhu G, Wang S, Chen J, Wang Z, Liang X, Wang X, Jiang J, Lang J, Li L. Long noncoding RNA HAS2-AS1 mediates hypoxia-induced invasiveness of oral squamous cell carcinoma. Mol Carcinog. 2017;56:2210-22.

18. Chen IC, Chiang WF, Huang HH, Chen PF, Shen YY, Chiang HC. Role of SIRT1 in regulation of epithelial-to-mesenchymal transition in oral squamous cell carcinoma metastasis. Mol Cancer. 2014;13:254.

19. Li D, Li L. MicroRNA3666 inhibits breast cancer cell proliferation by targeting sirtuin 7. Mol Med Rep. 2017:16:8493-500.

20. Lee HS, Jung W, Lee E, Chang H, Choi JH, Kim HG, Kim A, Kim BH. SIRT7, H3K18ac, and ELK4 Immunohistochemical expression in hepatocellular carcinoma. J Pathol Transl Med. 2016;50:337-44.

21. Li W, Sun Z, Chen C, Wang L, Geng Z, Tao J. Sirtuin7 has an oncogenic potential via promoting the growth of cholangiocarcinoma cells. Biomed Pharmacother. 2018;100:257-66.

22. Wei W, Jing ZX, Ke Z, Yi P. Sirtuin 7 plays an oncogenic role in human osteosarcoma via downregulating CDC4 expression. Am J Cancer Res. 2017; 7:1788-803.

23. Shi H, Ji Y, Zhang D, Liu Y, Fang P. MicroRNA-3666-induced suppression of SIRT7 inhibits the growth of non-small cell lung cancer cells. Oncol Rep. 2016;36:3051-7.

24. Tang X, Shi L, Xie N, Liu Z, Qian M, Meng F, Xu Q, Zhou M, Cao X, Zhu WG, et al. SIRT7 antagonizes TGF-beta signaling and inhibits breast cancer metastasis. Nat Commun. 2017:8:318.

25. Lai CC, Lin PM, Lin SF, Hsu CH, Lin HC, Hu ML, Hsu CM, Yang MY. Altered expression of SIRT gene family in head and neck squamous cell carcinoma. Tumour Biol. 2013;34:1847-54.

26. Vakhrusheva O, Braeuer D, Liu Z, Braun T, Bober E. Sirt7-dependent inhibition of cell growth and proliferation might be instrumental to mediate tissue integrity during aging. J Physiol Pharmacol. 2008;59(Suppl 9):201-12.

27. Palmirotta R, Cives M, Della-Morte D, Capuani B, Lauro D, Guadagni F, Silvestris F. Sirtuins and Cancer: role in the epithelial-Mesenchymal transition. Oxidative Med Cell Longev. 2016;2016:3031459. 
28. Yu H, Ye W, Wu J, Meng X, Liu RY, Ying X, Zhou Y, Wang H, Pan C, Huang $W$. Overexpression of sirt7 exhibits oncogenic property and serves as a prognostic factor in colorectal cancer. Clin Cancer Res. 2014;20:3434-45.

29. McGlynn LM, McCluney S, Jamieson NB, Thomson J, MacDonald Al, Oien K Dickson EJ, Carter CR, McKay CJ, Shiels PG. SIRT3 \& SIRT7: potential novel biomarkers for determining outcome in pancreatic Cancer patients. PLoS One. 2015;10:e0131344.

30. Barber MF, Michishita-Kioi E, Xi Y, Tasselli L, Kioi M, Mogtaderi Z, Tennen RI, Paredes S, Young NL, Chen K, et al. SIRT7 links H3K18 deacetylation to maintenance of oncogenic transformation. Nature. 2012;487:114-8.

31. Malik S, Villanova L, Tanaka S, Aonuma M, Roy N, Berber E, Pollack JR, Michishita-Kioi E, Chua KF. SIRT7 inactivation reverses metastatic phenotypes in epithelial and mesenchymal tumors. Sci Rep. 2015;5:9841.

32. Vakhrusheva O, Braeuer D Fau - Liu Z, Liu Z Fau - Braun T, Braun T Fau Bober E, Bober E. Sirt7-dependent inhibition of cell growth and proliferation might be instrumental to mediate tissue integrity during Aging.

33. Simic P, Williams EO, Bell EL, Gong JJ, Bonkowski M, Guarente L. SIRT1 suppresses the epithelial-to-mesenchymal transition in cancer metastasis and organ fibrosis. Cell Rep. 2013;3:1175-86.

34. Luo L, McGarvey P, Madhavan S, Kumar R, Gusev Y, Upadhyay G. Distinct lymphocyte antigens 6 (Ly6) family members Ly6D, Ly6E, Ly6K and Ly6H drive tumorigenesis and clinical outcome. Oncotarget. 2016;7:11165-93.

35. Goswami CP, Nakshatri H. PROGgeneV2: enhancements on the existing database. BMC Cancer. 2014;14:970

Ready to submit your research? Choose BMC and benefit from:

- fast, convenient online submission

- thorough peer review by experienced researchers in your field

- rapid publication on acceptance

- support for research data, including large and complex data types

- gold Open Access which fosters wider collaboration and increased citations

- maximum visibility for your research: over $100 \mathrm{M}$ website views per year

At BMC, research is always in progress.

Learn more biomedcentral.com/submissions 